\title{
Technical-Tactical Behaviors Analysis of Male and Female Judo Cadets' Combats
}

\author{
Bianca Miarka1,2*, Diego Ignácio Valenzuela Pérez ${ }^{3 *}$, Esteban Aedo-Muñoz ${ }^{2,4,5}$, \\ Lucas Oliveira Fernandes da Costa ${ }^{1}$ and Ciro José Brito ${ }^{2}$
}

\begin{abstract}
'Laboratory of Psychophysiology and Performance in Sports \& Combats, School of Physical Education and Sport, Federal University of Rio de Janeiro, Rio de Janeiro, Brazil, ${ }^{2}$ Laboratory of Physiological and Motor Analysis in Health and Performance of the Physical Education Department, Federal University of Juiz de Fora, Governador Valadares, Brazil, ${ }^{3}$ Escuela de Kinesiologia, Facultad de Salud, Universidad Santo Tomás, Santiago, Chile, ${ }^{4}$ Biomechanics Laboratory, Chilean High-Performance Center, Physical Activity, Sport and Health Sciences Laboratory, Universidad de Santiago de Chile, Santiago, Chile, ${ }^{5}$ Physical Education Department, Universidad Metropolitana de Ciencias de la Educación, Santiago, Chile
\end{abstract}

\section{OPEN ACCESS}

Edited by:

Miguel-Angel Gomez-Ruano, Polytechnic University of Madrid,

Spain

Reviewed by:

Raquel Hernández García,

University of Murcia, Spain

Ewa Polak,

Rzeszow University of Technology,

Poland

Coral Falco,

Western Norway University of Applied

Sciences, Norway

*Correspondence:

Bianca Miarka

miarkasport@hotmail.com

Diego Ignácio Valenzuela Pérez diegovalenzuela@santotomas.cl

Specialty section:

This article was submitted to

Movement Science and Sport

Psychology,

a section of the journal

Frontiers in Psychology

Received: 19 March 2020

Accepted: 25 May 2020

Published: 19 June 2020

Citation:

Miarka B, Pérez DIN

Aedo-Muñoz E, da Costa LOF and Brito CJ (2020) Technical-Tactical Behaviors Analysis of Male and Female Judo Cadets' Combats.

Front. Psychol. 11:1389.

doi: 10.3389/fpsyg.2020.01389
This brief research report showed technical-tactical behaviors of male and female judo cadets during combats, comparing the frequency and time of judo combat actions, techniques and penalties. The data was composed for 3,240 sequential technical-tactical behavior analysis from 108 female and 300 male cadet combats recorded of public judo championships. Combat, standing combat moments, approach action, gripping action, attack, groundwork actions and pause moment were observed and determinant technical-tactical behaviors (frequencies of actions, penalties and type of attacks) analysis were done with FRAMI software, followed by Mann-Whitney and Student's $t$-test, $p \leq 0.05$. Our main results indicated that male cadets with 58.66s \pm 50.26 s demonstrated longer gripping action than female with $38.44 \mathrm{~s} \pm 30.44 \mathrm{~s}$, as standing combat (tachi-waza) had differences between male with $96.8 \mathrm{~s} \pm 72 \mathrm{~s}$ and female athletes with $75.85 \mathrm{~s} \pm 56.97 \mathrm{~s}$. Moreover, male cadets had higher sacrifice techniques (sutemi-waza) actions than female athletes. This information could be used to a best performance associated with "psyching-up" as much as it could be used on physical training and technical-tactical ability of female and male cadets.

Keywords: motor control, sport psychology, task performance and analysis, martial arts, gender

\section{INTRODUCTION}

Gender has been recognized as a major difference of combat behavior over the effect of anthropometric and physical condition features related to the genetic and hormonal variances (Koyama et al., 2019; Schor et al., 2019). Some authors have suggested that gender differences in behavior conditions during combats may increase in judo (Suzuki et al., 2019); other have studied physiological sex differences in combat sports, but with limited behavior data. However, men and women differences should first be studied through the complete panel of quantifiable behavior sequential analysis. Numerous coaches around the world have experienced that best performance associated with psyching-up strategies on the physical and technical-tactical training (Tod et al., 2015). Cognitive approaches are consistently associated with behavior analysis and improved performance (results range from 61 to 65\%) (Tod et al., 2015). However, there is a lack of information for the practical application of sport psychology methods to acquire and retain new skills in cadet's judo, as well as to modify behaviors which reduce performance. 
For instance, technical-tactical analysis in judo emphasis on recognizing sequential behavior arrangements, often denoted to as "indicators," in the competitive situation (Brito et al., 2017). The performance in judo is associated with the ability of a male or female athlete to execute specific actions at the correct moment during each combat moment while quickly adapting to the constantly fluctuating combat environment to the next action (Brito et al., 2017). Technical-tactical behavior analysis have been well-described in judo, verifying match demands (Coswig et al., 2018), techniques and muscle group specific torque production (Lech et al., 2015). Specifically, during female and male judo adult's competition, technical-tactical actions of different technical-tactical behaviors and pause strategies are highly varied and the associations between these variables together describe a judo's system of attack or fighting style (López Díaz-de-Durana et al., 2018). Despite this important finding about the senior age class, until now, it is not very clear whether there are technical-tactical behavior differences between female and male youngers.

Particular behavior patterns that could determine different male and female cadet performance, considering maturational state and age effects (Fukuda, 2015), such as in senior, the approach trying to grip, gripping behaviors (kumi-kata), and scored attacks with different combinations (Coswig et al., 2018). Pinelopi Stavrinou et al. (2017) indicated a high influence that several judo bouts have in perceived exertion and cardiovascular system, highlighting the anaerobic pathway as the main metabolic involvement during four simulated judo combats, with about 11 attacks on standing combat (tachi-waza) and one attack on groundwork combat (ne-waza) per combat. Preceding authors demonstrated the use of specific effective tactical behaviors in high level cadet judo athletes, as re-gripping patterns during the gripping action (Ito et al., 2019). Despite this important finding, little is known about the differences between men and women cadets in relation to the behaviors of the gripping action. Female judo cadets training has largely been ignored throughout the youth training programs and studies about behavior judo analysis. Cadets judo combat research, programming and interventions have focused almost exclusively on men athletes with little attempt to understand the gender specific needs of women younger practitioners. Therefore, individual training programs could be adjusted for male and female judo athletes with consideration for the technical-tactical behavior and temporal structure of the competitive environment (Sterkowicz-Przybycien et al., 2017).

Regarding senior class, attacks from the same gripping preparation increase probabilities of resulting in a successful score for both male and female athletes (Muddle et al., 2017). The gripping moment seem to be associated with sequential actions during judo combat and critical indicators (Calmet et al., 2006). Other research indicated that percentage of total time spent by beginners with $20 \mathrm{~h}$ of practice and judo black-belt experts gripping the opponent's judogi with both hands, attacking and throwing were $\sim 90$ and $\sim 25 \%$ respectively, indicating differences associated with accumulated time of practice (Calmet et al., 2010). This phenomenon has not been investigated in cadet's judo championships, considering female and male combats. A full literature review did not produce any research addressing the ability to integrate gripping time and judo behaviors based on juvenile actions during competitions. Comparing genders.

In the same way, the gripping demand that occurs in the senior class shows specifics tactical arrangements with stances opposite from their opponents (kenka-yotsu) to preserve a defensive situation preceding to attack or counter-attack (Ito et al., 2019). A recent research suggested that motor action analysis in judo be conducted with consideration for sex modifications in senior combat actions with a wide range of interconnected behavior components (Sterkowicz-Przybycien et al., 2017), such as the approach action (Sterkowicz-Przybycien et al., 2017), attack with the type of technique (Anthierens et al., 2019), groundwork attempts (Brito et al., 2017) and pause phase analysis (SterkowiczPrzybycien et al., 2017). Currently, the specific behaviors of each gender in cadet class remain unknown; this knowledge is essential to target technical-tactical training, considering each combat phase. Therefore, the purpose of the present research was to report technical-tactical behaviors, comparing occurrences and respective time of judo combat actions, scores and penalties used by female and male cadet athletes during championships.

\section{METHODS}

\section{Sample}

The data was composed for 3,240 sequential technical-tactical behavior analysis from 108 female and 300 male cadet combats recorded of public judo championships. In order to be included, each video had to be with a minimal standard definition of 480/60i and taken from a landscape assessment of the entire competition area. The competitive bouts were evaluated following previously outlined protocols (López Díazde-Durana et al., 2018), from four local competitions. The VirtualDub Program 1.8.6(2) was used to fragment and edit records and the validated judo match analysis, Frami ${ }^{\circledR}$ software (Miarka et al., 2011), was utilized to make the technical-tactical behavior analysis; the present documentary research guaranteed confidentiality and anonymity by replacing the athletes' personal ID. All participants had previous experience ( $>2$ years) with official judo events (range level: local to national), rules and procedures (International Judo Federation (IJF), 2018). No modifications were made in the judo training (range frequency: two to five times/week, range time: three to $12 \mathrm{~h}$ /week) nutritional or hydration status of participants, following preceding protocols (Antonietto et al., 2019). There are no ethical problems in investigating data of public events, as disposed by prior protocols (Sterkowicz-Przybycien et al., 2017). Present research was earlier approved by the local Ethics and Research Committee, following WMA Declaration of Helsinki.

\section{Technical-Tactical Behaviors Analysis and Reliability Testing}

A behavior analysis of combat states was performed according to each frequency of occurrence and time, then normalized and presented by the combat and pause ratio. The analysis 
was defined in periods of the combat actions: approach, gripping, attack, groundwork and pause, following preceding reports (Sterkowicz-Przybycien et al., 2017). So, the approach was well-defined as the moment between the indication from the referee announcing the start of contest (hajime) and the realization of the grip (kumi-kata). During this moment, participants do not have any contact between them (Calmet et al., 2010). Gripping action was demarcated as the moment between the accomplishment of the grip (kumi-kata) and the breaking of interaction with the adversary judogi (Miarka et al., 2011). Attack was demarcated as the preliminary breaking balance of the adversary ( $k u z u s h i)$ and the preparatory changes of the body, particularly of the feet, completed prior to the attack (tsukuri) and the final displacement, trying to throw the opponent (kake) (Miarka et al., 2015). The groundwork combat was the moment where one or both fighters executed groundwork actions (ne-waza), according to previous protocols (Sterkowicz-Przybycien et al., 2017). The pause was the moment between the signal for contest pause ("matte") and the signal to restart the contest, with a voice command (hajime). The present study did not verify the "sono-mama" and "yoshi" commands halting and restarting the combat, which would also have been analyzed as recovery if they had had happened (Sterkowicz-Przybycien et al., 2017). Lastly, combat action or phase was the moment between the order from the order to initiate the combat (hajime), the order to stop the combat (matte), and the order to finish the combat (soremade), according to previous protocols (SterkowiczPrzybycien et al., 2017). Figure 1 demonstrates the behavior analysis model. Attacks that have been prohibited by rules have not been documented.
The index and classification of Kappa values of technical-tactical behavior analysis used in the present study for Inter-expert and Intra-expert measurements were 0.74 and 0.82, classified as "Strong" and "Almost perfect" for Approach Action, 0.45 and 0.96, classified as "Moderate" and "Almost perfect" for Gripping Action, 0.52 and 0.97, classified as "Moderate" and "Almost perfect" for Attack Action, 0.84 and 0.90, classified as "Almost perfect" and "Almost perfect" for Groundwork Actions and 0.91 and 0.99, classified as "Almost perfect" and "Almost perfect" for Pause Phase. The classification of nage-waza (throwing techniques) and katame-waza (groundwork techniques) attacks considered the nage-waza techniques classification of Kodokan, correspondingly in the procedure of the four throwing types: te-waza (arm techniques) with 0.75 and 1.00 classified as substantial, ashiwaza (leg/foot technique) with 0.97 and 1.00 classified as "Almost Perfect," koshi-waza (hip technique) with 1.0 and 1.00 classified as "Almost Perfect," and sutemi-waza (sacrifice technique) with 0.96 and 0.95 classified as "Almost Perfect," Mean-Whitney comparisons did not show effects between intra or inter measurements, $p \leq 0.05$, following preceding indications (Dal Bello et al., 2019).

\section{Statistical Analysis}

The SPSS 20.0 for Windows was used to obtain descriptive and statistical inferences. Descriptive statistics were demonstrated as mean and standard deviation (SD), and Independent T-tests conducted to compare time-motion variables. While frequencies were showed as median, mean [25th percentile; 75 th percentile] values and Mann-Whitney tests were used to compare combat phase and technical-tactical frequencies between male and female

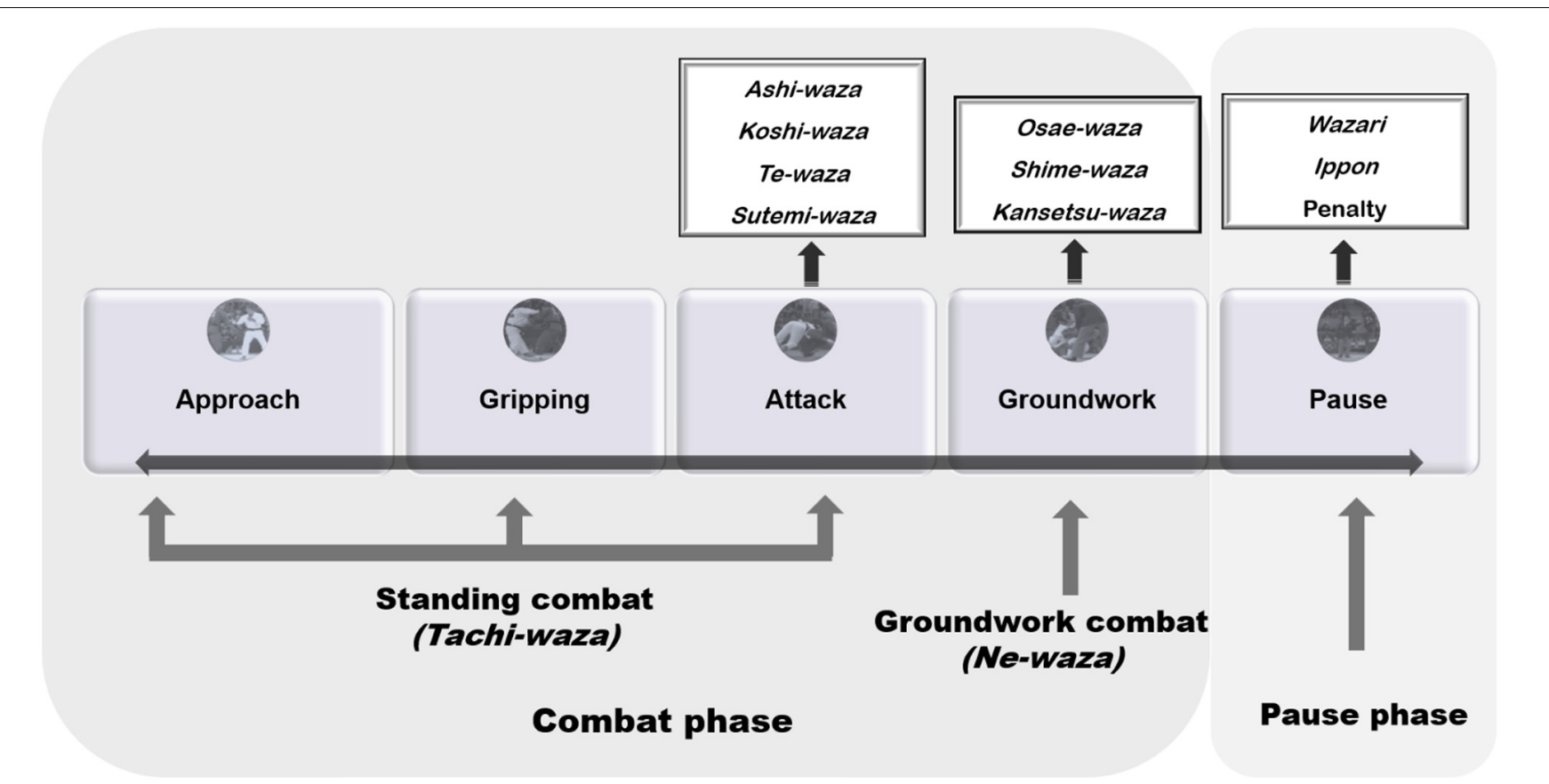

FIGURE 1 | Behavior judo combat model analysis. 
judo cadets. Effect size was verified following preceding reports (Brito et al., 2017), considering the significance level of $p \leq 0.05$.

\section{RESULTS}

Comparisons of time-motion analysis of judo actions between male and female cadets are in Table $\mathbf{1}$.

Male cadet judo athletes presented longer gripping action than female fighters and, longer tachi-waza moment.

Table 2 shows technical-tactical behaviors of cadets during judo championships.

There was no significant difference for any comparison $(p>0.05)$.

\section{DISCUSSION}

Despite the amount of technical-tactical behaviors studies in judo, few evaluated cadet athletes. Technical-tactical behavior studies in this age group can help coaches to properly train and prepare their athletes for competitions. Therefore, the present study compared technical-tactical behavior analysis between male and female cadet judo combats. It was the first time, for the best of our knowledge, that the combination of technical-tactical actions that was presented comparing men and women cadets. The main results demonstrated a main difference between groups, male cadets with longer gripping action, tachi-waza moment and higher sutemi-waza frequencies than female athletes. This data can be used to

TABLE 1 | Comparisons of behavior judo combat analysis of female versus male cadets (time).

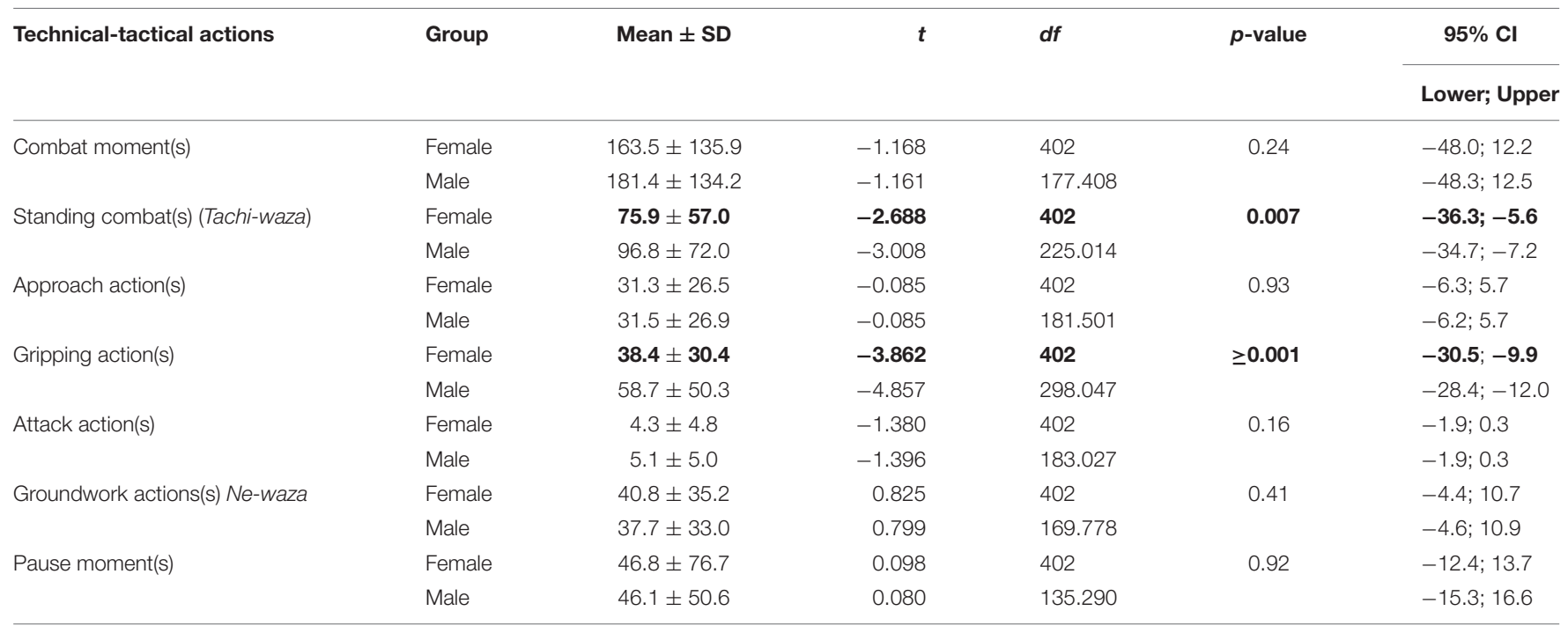

Variables with significant difference are in bold.

TABLE 2 | Comparisons of technical-tactical behaviors judo combat of female versus male cadets (frequencies).

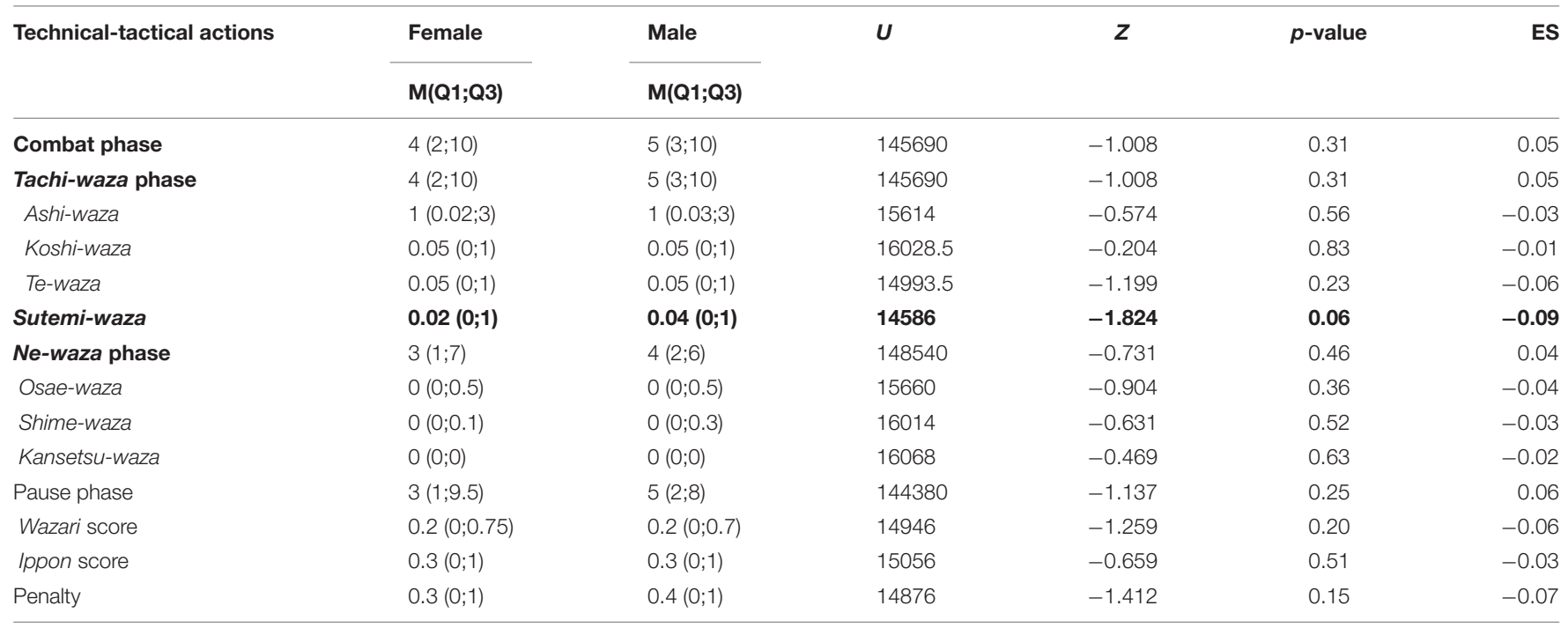

Variables with significant difference are in bold. 
create cognitive strategies on contextual and physical training (Tod et al., 2015). Mental strategies are consistently connected with $\sim 65 \%$ of improved performance (Tod et al., 2015). Present judo behavior model and results had emerged to bridge the gap between research and mental strategy practices for competitive events and rehabilitation, as technical and tactical behavior models associated with youngers were not available in scientific studies.

Regarding present results, the longer gripping moment and the use of both collar grip was recurrent in defensive situations, as it could maintain a high space control over the opponent, but it reduced opponent's imbalance possibilities (kuzushi) and, consequently affected the execution of different techniques and biomechanical actions during collect data analysis. Cadet judo athletes demonstrated $50-60 \%$ of shorter tachi-waza moment than pre-juvenile, juvenile, junior and senior judokas at the same level (Miarka et al., 2012). In contrast, groundwork actions and pause moment analysis presented similar results when compared with male and female pre-juvenile, juvenile, junior and female senior judo athletes i.e., $42 \pm 33 \mathrm{~s}$ and $41 \pm 31 \mathrm{~s}, 37 \pm 26 \mathrm{~s}$ and $35 \pm 26 \mathrm{~s}$, and $33 \pm 26 \mathrm{~s}$ and $41 \pm 35$ s and $36 \pm 31$ s (Miarka et al., 2014) - all them shorter than male senior athletes with $50 \pm 37$ s (Miarka et al., 2012). This could be clarified by considering the modifications in the rules that were changed in order to make judo a more attractive sport to the public, consequently lower groundwork behaviors were observed in specific weight divisions (Sterkowicz-Przybycien et al., 2017). Furthermore, modifications in rules over the last years have proposed to enhance the dynamic of the combat, which may have impacted behavioral strategies, injuries frequencies and pause moments (Stephenson and Rossheim, 2018).

Preceding authors indicated that sport specialization of judo cadets was not related with success across an athletic career, finding indicated that less than $7 \%$ of the athletes repeated their initial state-level competitive outcomes after 10 years of judo competition (Julio et al., 2011). indeed, the evidence is contrary to this hypothesis as the pre-juvenile male age class had a lower percentage of medal winners in the last year of the 10 years follow-up, and male and female pre-juvenile age classes had a lower percentage of medal winners in the last 3 years of this follow-up than the grand mean (Julio et al., 2011). Therefore, the early specialization procedure in judo is not likely to be related with success in senior age class, and programs directed to talent promotion should emphasis the development of motor skills, contextual training and mental development than the competitive outcome (Julio et al., 2011). Concerning this programs, present research contributes with essential information for the development of motor skills, as well as the mental and physical training of youth practitioners, according to their match references, avoiding the use of senior match situations.

Our evaluated athletes showed a higher tendency to take with the traditional grip, right handgrip on the collar and left handgrip on the sleeve (migi-kumi-katá) and present a higher volume of ashi-waza - this type of technique is used twice as often when compared to other types during local/regional cadet judo championship. Previous studies reported a predominance of te-waza during high level senior athletes in the London Olympic Games, but with specific differences in the techniques applied (Sterkowicz-Przybycien et al., 2017). While authors observed higher use of ashi-waza in junior versus senior of state and national level female athletes (Kamitani et al., 2017). Male cadets presented higher frequencies of sacrifice techniques than female. The use of sutemi-waza has been described to happen more often in senior compared to junior athletes (Miarka et al., 2014) and may be chosen during male cadets' championship due to greater scoring efficiency. Ashi-waza techniques could not result in higher scores, but it is known the higher attack volume can result in competitive advantage since will result in a penalty to the opponent (Brito et al., 2017), a factor that should be considered by coaches when designing the training and preparing competitive strategies of cadets.

Groundwork combat did not demonstrate higher predominance in recreational cadets' level, while for high level female athletes, osae-waza was determinant as winning factor (Miarka et al., 2017). In fact, groundwork motor actions were linked to effective actions of $20 \%$ of all attempts to attack during the Olympic Games (SterkowiczPrzybycien et al., 2017). In a previous study, senior judo matches presented a greater use of osae-waza, shime-waza and kansetsu-waza (Sterkowicz-Przybycien et al., 2017). In senior high-level athletes, the osae-waza increased the performance probability when combined with judo techniques that implicate rotation, for instance morote-seoi-nage, and sacrifice throws (sutemi-waza), for example tomoe-nage (Miarka et al., 2017). Previous findings indicated that the use of ahi-waza with Arm/foot lever to realize a fast transition to the groundwork could be responsible of $\sim 60 \%$ of the winning cases affecting positively the osae-waza (Miarka et al., 2017).

A potential limitation of behavior analysis is the consistency of the data achievement procedure, or the researcher's capability to reproduce the detected behavior once measurement is repeated (Coswig et al., 2018). Large variations in the combat moment, frequency and mean duration of actions measured during reliability analyses can affect inter-observer consistency (Coswig et al., 2018). The observational-descriptive approach implemented in the current investigation may limit extrapolation of the results. Furthermore, cadet athletes likely produce dissimilar physical and physiological effects for a particular combat behavior than senior judo athletes (Coswig et al., 2018). During the present behavior analysis, weight divisions of female and male cadets demonstrated great temporal similarities, except for female half-heavyweight and heavyweight categories, in which tachi-waza (i.e., approach/gripping phases) was longer than half-lightweight division; however, without significant effects in the behavior frequencies, score or penalties for male and female younger judo athletes. This also indicates the need for future further research to verify how changes in regulation affect the cadet combat and their respective weight divisions and technical-tactical actions. 


\section{CONCLUSION}

Our results demonstrated the differences between men and women judo cadet athletes - with longer gripping action and tachi-waza preference of male than female combats. These differences among groups need to be reflected in judo physical and mental training practices. Our findings emphasize the importance of accounting the frequency and time of technical-tactical behaviors and the singularities of each gender. Therefore, psychologists and coaches can make extensive use of behavior analysis during championships and data collection measures to deliver advantageous feedback about each combat action while developing systematic methods of mental and physical technical-tactical training, focusing on system of attack variations, using groundwork attacks (katame-waza), avoiding longer time with the same gripping behavior, which negatively influence the control over the opponent and it could increase the chance to receive penalties during cadets championships.

\section{REFERENCES}

Anthierens, A., Olivier, N., Thevenon, A., and Mucci, P. (2019). Trunk muscle aerobic metabolism responses in endurance athletes, combat athletes and untrained men. Int. J. Sports Med. 40, 434-439. doi: 10.1055/a-0856-7207

Antonietto, N. R., Bello, F. D., Carrenho Queiroz, A. C., Berbert de Carvalho, P. H., Brito, C. J., Amtmann, J., et al. (2019). Suggestions for professional mixed martial arts training with pacing strategy and technical-tactical actions by rounds. J. Strength Cond. Res. doi: 10.1519/JSC.0000000000003018 [Epub ahead of print].

Brito, C. J., Miarka, B., de Durana, A. L. D., and Fukuda, D. H. (2017). Home advantage in judo: analysis by the combat phase, penalties and the type of attack. J. Hum. Kinet. 57, 213-220. doi: 10.1515/hukin-2017-0062

Calmet, M., Miarka, M., and Franchini, E. (2010). Modeling of grasps in judo contests. Int. J. Perf. Anal. Sport 10, 229-240. doi: 10.1080/24748668.2010. 11868518

Calmet, M., Trezel, N., and Ahmaidi, S. (2006). Survey of system of attacks by judoka in regional and interregional matches. Percept. Mot. Skills 103, 835-840. doi: 10.2466/pms.103.3.835-840

Coswig, V. S., Gentil, P., Bueno, J. C., Follmer, B., Marques, V. A., and Del Vecchio, F. B. J. P. (2018). Physical fitness predicts technical-tactical and timemotion profile in simulated judo and brazilian jiu-jitsu matches. Cell 6:e4851. doi: $10.7717 /$ peerj.4851

Dal Bello, F., Aedo-Muñoz, E., Brito, C. J., and Miarka, B. (2019). Performance analysis and probabilities by gender in judo: combat phases, techniques and biomechanical levers. Facta Univer. Ser. 17, $135-148$.

Fukuda, D. H. (2015). Analysis of the relative age effect in elite youth judo athletes. Int. J. Sports Physiol. Perform. 10, 1048-1051. doi: 10.1123/ijspp.20140463

International Judo Federation (IJF) (2018). News About Judo Competition. Available online at: https://www.ijf.org/news/list?tag[0]=competition

Ito, K., Hirose, N., and Maekawa, N. (2019). Characteristics of re-gripping techniques preceding scored throws in international-level judo competition. Cent. Eur. J. Sport Sci. Med. 25:7.

Julio, U. F., Takito, M. Y., Mazzei, L., Miarka, B., Sterkowicz, S., and Franchini, E. (2011). Tracking 10-year competitive winning performance of judo athletes across age groups. Percept. Mot. Skills 113, 139-149. doi: 10.2466/05.10.11.PMS. 113.4.139- 149

Kamitani, T., Malliaropoulos, N. G., Omiya, M., Otaka, Y., Inoue, K., and Onidani, N. (2017). On the way to the Tokyo Summer Olympic Games (2020). Prevention of severe head and neck injuries in judo: it's time

\section{DATA AVAILABILITY STATEMENT}

The raw data supporting the conclusions of this article will be made available by the authors, without undue reservation, to any qualified researcher.

\section{ETHICS STATEMENT}

Present research was earlier approved by the local Ethics and Research Committee, following WMA Declaration of Helsinki. Written informed consent to participate in this study was provided by the patient/participants' or patient/participants' legal guardian/next of kin where required.

\section{AUTHOR CONTRIBUTIONS}

All authors listed have made a substantial, direct and intellectual contribution to the work, and approved it for publication.

for action. Br. J. Sports Med. 51, 1581-1582. doi: 10.1136/bjsports-2017097681

Koyama, K., Hirokawa, M., Yoshitaka, Y., and Yamauchi, J. (2019). Toe flexor muscle strength and morphological characteristics of the foot in judo athletes. Int. J. Sports Med. 40, 263-268. doi: 10.1055/a-07966679

Lech, G., Chwala, W., Ambrozy, T., and Sterkowicz, S. (2015). Muscle torque and its relation to technique, tactics, sports level and age group in judo contestants. J. Hum. Kinet. 45, 167-175. doi: 10.1515/hukin-20150017

López Díaz-de-Durana, A., Bello, F. D., Brito, C. J., and Miarka, B. (2018). High level performance in world judo circuit: notational analyzes of combat phase by weight categories. J. Hum. Sport Exerc. 13, S329-S338.

Miarka, B., Branco, B. H. M., Vecchio, F. B. V. D., Camey, S., and Franchini, E. (2015). Development and validation of a time-motion judo combat model based on the Markovian Processes. Int. J. Perf. Anal. Sport 15, 315-331. doi: 10.1080/24748668.2015.11868795

Miarka, B., Cury, R., Julianetti, R., Battazza, R., Julio, U. F., Calmet, M., et al. (2014). A comparison of time-motion and technical-tactical variables between age groups of female judo matches. J. Sports Sci. 32, 1529-1538. doi: 10.1080/ 02640414.2014 .903335

Miarka, B., Hayashida, C. R., Julio, U. F., Calmet, M., and Franchini, E. (2011). Objectivity of FRAMI-software for judo match analysis. Intern. J. Perform. Anal. Sport 11:11. doi: 10.1080/24748668.2011.1186 8546

Miarka, B., Panissa, V. L., Julio, U. F., Del Vecchio, F. B., Calmet, M., and Franchini, E. (2012). A comparison of time-motion performance between age groups in judo matches. J. Sports Sci. 30, 899-905. doi: 10.1080/02640414.2012. 679675

Miarka, B., Sterkowicz-Przybycien, K., and Fukuda, D. H. (2017). Evaluation of Sex-specific movement patterns in judo using probabilistic neural networks. Mot. Control 21, 390-412. doi: 10.1123/mc.2016-0007

Muddle, T. W. D., Fukuda, D. H., Wang, R., Riffe, J. J., Church, D. D., Beyer, K. S., et al. (2017). Effects of a 10-week introductory judo course on postural control during a bilateral reactionary gripping task. Mot. Control 21, 373-389. doi: $10.1123 / \mathrm{mc} .2016-0040$

Pinelopi Stavrinou, S., Argyrou, M., and Hadjicharalambous, M. (2017). Physiological and metabolic responses during a simulated judo competition among cadet athletes. Int. J. Perform. Anal. Sport 16:11.

Schor, B., Silva, S. G. D., Almeida, A. A., Pereira, C. A. B., and Arida, R. M. (2019). Plasma brain-derived neurotrophic factor is higher after combat training 
(Randori) than incremental ramp test in elite judo athletes. Braz. J. Med. Biol. Res. 52:e8154.

Stephenson, C., and Rossheim, M. E. (2018). Brazilian Jiu Jitsu, Judo, and mixed martial arts injuries presenting to United States emergency departments, 2008-2015. J. Prim. Prev. 39, 421-435. doi: 10.1007/s10935-0180518-7

Sterkowicz-Przybycien, K., Miarka, B., and Fukuda, D. H. (2017). Sex and weight category differences in time-motion analysis of elite judo athletes: implications for assessment and training. J. Strength Cond. Res. 31, 817-825. doi: 10.1519/ jsc. 0000000000001597

Suzuki, Y., Ikeda, A., Maruyama, K., Sakamoto, N., Wada, H., and Tanigawa, T. (2019). The association between obesity and hyperactivity/anxiety among elementary school students in Japan. Int. J. Behav. Med. 27, 79-86. doi: 10.1007/s12529-019-09 $827-\mathrm{x}$
Tod, D., Edwards, C., McGuigan, M., and Lovell, G. (2015). A Systematic review of the effect of cognitive strategies on strength performance. Sports Med. 45, $1589-1602$.

Conflict of Interest: The authors declare that the research was conducted in the absence of any commercial or financial relationships that could be construed as a potential conflict of interest.

Copyright (c) 2020 Miarka, Pérez, Aedo-Muñoz, da Costa and Brito. This is an open-access article distributed under the terms of the Creative Commons Attribution License (CC BY). The use, distribution or reproduction in other forums is permitted, provided the original author(s) and the copyright owner(s) are credited and that the original publication in this journal is cited, in accordance with accepted academic practice. No use, distribution or reproduction is permitted which does not comply with these terms. 